\title{
Comparison of Numerical Methods for the Calculation of Two-Dimensional Turbulence
}

\author{
By G. L. Browning and H.-O. Kreiss* \\ Dedicated to Professor Eugene Isaacson on the occasion of his 70 th birthday
}

\begin{abstract}
The estimate derived by Henshaw, Kreiss, and Reyna for the smallest scale present in solutions of the two-dimensional incompressible Navier-Stokes equations is employed to obtain convergent pseudospectral approximations. These solutions are then compared with those obtained by a number of commonly used numerical methods.

If the viscosity term is deleted and the energy in high wave numbers removed by setting the amplitudes of all wave numbers above a certain point in the spectrum to zero, the "chopped" solution differs considerably from the convergent solution, even at early times. In the case that the regular viscosity is replaced by a hyperviscosity term, i.e., the square of the Laplacian, we also derive an estimate for the smallest scale present. If the coefficient of hyperviscosity is chosen so that the spectrum of the hyperviscosity solution disappears at the same point as for the regular viscosity solution, the hyperviscosity solution is also completely different from the convergent solution. If we "tune" the hyperviscosity coefficient, then the solutions are similar in amplitude or phase, but not both.

The solution obtained by a second-order difference method with twice the number of points as the pseudospectral model, or a fourth-order difference method with the same number of points as the pseudospectral model, is essentially identical to the convergent solution. This is reasonable since most of the energy of the solution is contained in the lower part of the spectrum.
\end{abstract}

1. Introduction. Three-dimensional turbulence simulations based on the incompressible Navier-Stokes equations require an inordinate amount of computer power. Therefore, many numerical studies of turbulence (Lilly [9], Fox and Orszag [5], Herring et al. [7], Fornberg [4]) have been based on the two-dimensional version of these equations, which we briefly discuss in Section 2. Recently, an upper bound on the number of waves that are necessary to resolve solutions of the twodimensional equations with a given kinematic viscosity have been derived (Henshaw et al. [6]). In Section 3 we discuss the estimate and use it to obtain convergent numerical solutions. Since we have convergent numerical solutions of the twodimensional equations, we can compare a number of numerical methods that have been used for the computation of two-dimensional turbulence.

To remove energy in high wave numbers, a number of numerical models have incorporated "chopping", i.e., the models set the amplitude of all waves above a fixed point in the spectrum to zero periodically in time (e.g. Fornberg [4]). There is no analytic method to determine at which point to chop the spectrum or how often

Received April 28, 1988.

1980 Mathematics Subject (lassification (1985 Revision). Primary 35B30, 35A40.

*The work of this author was supported in part by the National Science Foundation under Grant DMS-8312264 and by the Office of Naval Research under Contract N-00014-83-K-0422. 
to perform the chopping, so it must be done by numerical investigation. Section 4 compares convergent solutions for a fixed kinematic viscosity with those obtained from chopping the spectrum in order to explore the robustness of the chopping method.

In an attempt to use a smaller effective kinematic viscosity, some models have employed higher-order dissipation mechanisms which damp the high wave numbers more selectively (McWilliams [10]). In Section 5 we derive estimates similar to those obtained by Henshaw et al. [6] for the case where the dissipation is the square of the Laplacian. A comparison of convergent numerical solutions from regular and hyperviscosity cases indicates differences and similarities that can be expected when using different forms of viscosity.

Early turbulence models employed finite difference methods in both space and time (e.g. Lilly [9]). More recently, the pseudospectral method has been employed for increased spatial accuracy (Fox and Orszag [5], Brachet et al. [2]). Section 6 compares solutions obtained from second- and fourth-order finite difference spatial methods with convergent solutions from the pseudospectral spatial method. This comparison is very important since finite difference methods for the initial-boundary problem are easier to develop and analyze, but conceivably might be less accurate than pseudospectral methods.

2. Mathematical and Numerical Models. A commonly used mathematical model of turbulence is the incompressible Navier-Stokes system of equations which in Cartesian coordinates $(x, y, z)$ can be written as

$$
\begin{aligned}
& \frac{D \mathbf{V}}{D t}+\nabla \phi=\nu \nabla^{2} \mathbf{V} \\
& \nabla \cdot \mathbf{V}=0,
\end{aligned}
$$

where $t$ is time, $\mathbf{V}=(u, v, w)$ is the velocity, $\phi$ is the pressure $p$ divided by the constant density $\rho, \nu$ is the kinematic viscosity (the viscosity $\mu$ divided by the density), and $\frac{D}{D t}$ is the substantial derivative defined as

$$
\frac{D \mathbf{V}}{D t}=\frac{\partial \mathbf{V}}{\partial t}+(\mathbf{V} \cdot \nabla) \mathbf{V}=\frac{\partial \mathbf{V}}{\partial t}+\nabla\left(V^{2} / 2\right)-\mathbf{V} \times \nabla \times \mathbf{V}
$$

Define the vorticity $\mathbf{Z}$ as

$$
\mathbf{Z}=\nabla \times \mathbf{V}
$$

Applying the curl operator to (2.1a) and (2.2) and using standard vector identities (Book [1]), we obtain the following equations for $\mathbf{Z}$ and $\mathbf{V}$ :

$$
\begin{aligned}
& \frac{\partial \mathbf{Z}}{\partial t}+(\mathbf{V} \cdot \nabla) \mathbf{Z}-\mathbf{V}(\nabla \cdot \mathbf{Z})+(\mathbf{Z} \cdot \nabla) \mathbf{V}=\nu \nabla^{2} \mathbf{Z} \\
& \nabla^{2} \mathbf{V}=-\nabla \times \mathbf{Z}
\end{aligned}
$$

and system (2.3) is equivalent to system (2.1).

In this paper we will only study the two-dimensional version of (2.3), i.e., we will assume $\mathbf{V}=(u, v, 0)$ and $\mathbf{Z}=(0,0, \varsigma)$, where $\varsigma$ is only a function of $x$ and $y$. For this case the last two terms on the left-hand side of $(2.3 \mathrm{a})$ are zero, and we can 
write the system as

$$
\begin{aligned}
& \varsigma_{t}+u \varsigma_{x}+v \varsigma_{y}=\nu \nabla^{2} \varsigma \\
& \nabla^{2} u=-\varsigma_{y} \\
& \nabla^{2} v=\varsigma_{x} .
\end{aligned}
$$

As the divergence $\delta=u_{x}+v_{y}$ is zero, we can also write (2.4a) in the form

$$
\zeta_{t}+(u \zeta)_{x}+(v \zeta)_{y}=\nu \nabla^{2} \zeta
$$

We consider system (2.4) in the region $R=\{(x, y) \mid 0 \leq x \leq 2 \pi, 0 \leq y \leq 2 \pi\}$ and assume the solutions of interest are periodic in $x$ and $y$. The initial conditions will be chosen so that the initial energy spectrum has one of several specified decay rates, which we will describe in the following section.

We approximate solutions of (2.4) by two different numerical methods. For either method we first approximate $R$ by the finite difference grid $G=\left\{\left(x_{i}, y_{j}\right) \mid x_{i}=\right.$ $\left.(i-3) \Delta s, 1 \leq i \leq 2 N+4 ; y_{j}=(j-3) \Delta s, 1 \leq j \leq 2 N+4\right\}$, where the grid spacing $\Delta s$ is determined as $\Delta s=\pi / N$ with $N$ a given power of two. The points outside of $R$ are used to implement the periodic boundary conditions for finite difference approximations in space. The time increment $\Delta t$ is chosen so that the CFL criterion is satisfied. We will use the standard notation for grid functions, i.e., $u_{i, j}^{n} \approx u\left(x_{i}, y_{j}, n \Delta t\right)$ and nominal subscripts or superscripts will not appear in the difference equations.

The first numerical model is based on the second-order leap-frog scheme in time and the pseudospectral method in space. Let $\hat{\zeta}$ denote the Fourier transform of $\zeta$ and let $\mathbf{k}^{T}=\left(k_{1}, k_{2}\right)$ be the index variable of the Fourier coefficients. We replace (2.4) by the approximation

$$
\begin{gathered}
\hat{\zeta}^{n+1}=\hat{\zeta}^{n-1}-2 \Delta t\left[i k_{1}(\hat{u} \zeta)+i k_{2}(\hat{v} \zeta)+.5 \nu\left(k_{1}^{2}+k_{2}^{2}\right)\left(\hat{\zeta}^{n+1}+\hat{\zeta}^{n-1}\right)\right] \\
-\left(k_{1}^{2}+k_{2}^{2}\right) \hat{u}=-i k_{2} \hat{\zeta} \\
-\left(k_{1}^{2}+k_{2}^{2}\right) \hat{v}=i k_{1} \hat{\zeta}
\end{gathered}
$$

Note that we are updating the Fourier coefficients of $\varsigma$ rather than the function $\varsigma$ itself. We have made an implicit approximation of the diffusion to maintain second-order accuracy in time, and it is much easier to solve the resulting system of equations in spectral space. Fourier interpolation is used to compute the derivatives (Kreiss and Oliger [8]). The transforms are performed using Temperton's implementation of the FFT.

The second numerical model is based on the second-order leap-frog method in time and second- or fourth-order finite difference approximations in space. In this case we replace $(2.4)$ by the approximation

$$
\left.\varsigma^{n+1}=\varsigma^{n-1}-2 \Delta t\left[D_{x}(u \zeta)+D_{y}(v \zeta)-\nu L \varsigma^{n-1}\right)\right],
$$

where $D_{x}\left(D_{y}\right)$ is the standard centered second- or fourth-order accurate finite difference approximation of $\frac{\partial}{\partial x}\left(\frac{\partial}{\partial y}\right)$ and $L$ is the standard second-order accurate approximation of the Laplacian operator. When using second-order approximations for $D_{x}$ and $D_{y}$, we can use $(2.5 \mathrm{~b})$ and $(2.5 \mathrm{c})$ to solve for $u$ and $v$, or a standard 
package which essentially solves the system

$$
\begin{aligned}
& -4(\Delta s)^{-2}\left[\sin ^{2}\left(.5 k_{1} \Delta s\right)+\sin ^{2}\left(.5 k_{2} \Delta s\right)\right] \hat{u}=-i(\Delta s)^{-1} \sin \left(k_{2} \Delta s\right) \hat{\zeta} \\
& -4(\Delta s)^{-2}\left[\sin ^{2}\left(.5 k_{1} \Delta s\right)+\sin ^{2}\left(.5 k_{2} \Delta s\right)\right] \hat{v}=i(\Delta s)^{-1} \sin \left(k_{1} \Delta s\right) \hat{\zeta}
\end{aligned}
$$

When using fourth-order approximations for $D_{x}$ and $D_{y}$, we use $(2.5 \mathrm{~b})$ and $(2.5 \mathrm{c})$ to solve for $u$ and $v$, so that the elliptic solver is at least fourth-order accurate. The approximation of the Laplacian for this case will be discussed in a later section.

3. Convergence Results. As system (2.4) is highly nonlinear, few analytic solutions are known, and most studies have involved the use of numerical models (e.g. Fornberg [4], McWilliams [10], Brachet et al. [2]). Recently, Henshaw et al. [6] have obtained precise estimates on the Fourier coefficients of solutions of (2.4) in the form

$$
\sup _{t \geq 1}|\hat{\zeta}| \leq\left[\frac{K_{q}(\alpha)|D \mathbf{u}|_{\infty}^{1 / 2+\alpha}}{\nu^{1 / 2+\alpha}|k|}\right]^{2 q},
$$

where $\alpha$ is a positive constant which can be chosen as small as desired, $q$ is any positive integer, $K_{q}(\alpha)$ is a constant depending on $q$ and $\alpha$ only, and

$$
|D \mathbf{u}|_{\infty}=\max _{t \geq 0}\left[\left|u_{x}(\cdot, t)\right|_{\infty},\left|u_{y}(\cdot, t)\right|_{\infty},\left|v_{x}(\cdot, t)\right|_{\infty},\left|v_{y}(\cdot, t)\right|_{\infty}\right]
$$

The maximum principle applied to $(2.4)$ shows that $|\zeta(\cdot, t)|_{\infty} \leq|\zeta(\cdot, 0)|_{\infty}$. We have normalized the initial data such that $|\zeta(\cdot, 0)|_{\infty} \leq 1$. Then it follows that $|D \mathbf{u}|_{\infty} \approx 1$. The constants $K_{q}(\alpha)$ can be estimated explicitly (see also Section 5 ). For $q \leq 3$ we obtain for all $\alpha>0$

$$
K_{q} \leq C_{q}\|\zeta(\cdot, 0)\|^{1 / q},
$$

where $\|\cdot\|$ denotes the $L_{2}$-norm and

$$
C_{1}=2^{1 / 2}, \quad C_{2}=3^{1 / 4}, \quad C_{3}=10^{1 / 6} .
$$

Therefore, for practical purposes, we can set $\alpha=0$ and $K_{q}=1$.

This estimate gives us the ability to determine very accurately the wave number above which the spectrum of the solution is negligible, i.e., for all $k$ with

$$
|k|>\nu^{-1 / 2}
$$

the spectral coefficients are essentially zero. We define the "changeover" wave number $k_{c}$ above which the spectrum is assured to be negligible as

$$
k_{c}=\nu^{1 / 2},
$$

and we expect that a numerical model that treats $k_{c}$ waves accurately in both directions will give us the correct solution of (2.4).

As a test of the estimate (3.3), we have run the pseudospectral model for the cases $\nu=10^{-4}$ and $\nu=10^{-5}$ with $32,64,128$, and 256 waves on a number of different initial conditions. Each of the initial conditions is chosen to have a different energy spectrum decay rate. The energy $E$ is defined as (McWilliams [10])

$$
E=\frac{1}{2} \frac{1}{(2 \pi)^{2}} \int_{R}\left(u^{2}+v^{2}\right) d x d y,
$$


and we can use Parseval's equality to write the energy in terms of the spectral coefficients of the velocity components or stream function:

$$
\begin{aligned}
E & =\frac{1}{2} \sum_{\mathbf{k}}\left(|\hat{u}|^{2}+|\hat{v}|^{2}\right)=\frac{1}{2} \sum_{\mathbf{k}}\left(\left|-i k_{2} \hat{\psi}\right|^{2}+\left|i k_{1} \hat{\psi}\right|^{2}\right) \\
& =\frac{1}{2} \sum_{\mathbf{k}}|\mathbf{k}|^{2}|\hat{\psi}|^{2}=\sum_{l=1}^{\infty} E_{l},
\end{aligned}
$$

where

$$
E_{l}=\frac{1}{2} \sum_{|\mathbf{k}| \in I_{l}}|\mathbf{k}|^{2}|\hat{\psi}|^{2}
$$

with $I_{l}=[l-.5, l+.5)$. In deriving (3.4), we have assumed that the initial conditions contain no energy in wave number $\mathbf{k}=0$.

The number of coefficients in $E_{l}$ is proportional to the area of the annulus with radius $l$ and width $\Delta l=1$. This implies that if we want an energy spectrum proportional to $l^{-r}$ for large $l$, then the stream function coefficients $\hat{\psi}$ must be proportional to $|\mathbf{k}|^{-(r+3) / 2}$ for large $l$. We chose four spectra used by previous authors. The amplitudes of the spectral coefficients for the four cases are given by

$$
\begin{aligned}
& |\hat{\psi}|=\left\{\begin{array}{ll}
C_{1}|\mathbf{k}|^{-2}, & |\mathbf{k}|<20.5 \\
0, & |\mathbf{k}| \geq 20.5
\end{array}\right\} \quad(r=1), \\
& |\hat{\psi}|=\left\{\begin{array}{ll}
C_{2}|\mathbf{k}|^{-3}, & |\mathbf{k}|<20.5 \\
0, & |\mathbf{k}| \geq 20.5
\end{array}\right\} \quad(r=3), \\
& |\hat{\psi}|=\left\{\begin{array}{ll}
C_{3}\left\{|\mathbf{k}|^{-2}\left[1+(|\mathbf{k}| / 6)^{4}\right]^{-1}\right\}^{1 / 2}, & |\mathbf{k}|<20.5 \\
0, & |\mathbf{k}| \geq 20.5
\end{array}\right\} \quad(r=3), \\
& |\hat{\psi}|=C_{4} .02^{1 / 2}|\mathbf{k}|^{-1} e^{-.125|k|^{2}} \quad\left(E_{l} \approx .02 k e^{-.25|k|^{2}}\right),
\end{aligned}
$$

and the phases in all cases are chosen as random numbers in the interval $[0,2 \pi]$. The initial distribution of energy (3.5) and (3.6) were used by Fornberg [4], (3.7) by McWilliams [10], and (3.8) by Brachet et al. [2]. Since we have assumed that $|D \mathbf{u}|_{\infty}=1$, the corresponding solutions must satisfy

$$
|\varsigma(\cdot, t)|_{\infty} \leq 2
$$

We chose the constants $C_{i}, i=1, \ldots, 4$, so that $|\varsigma(\cdot, 0)|_{\infty}=1$. Although we used all four initial conditions, we will only show the results for computations based on the initial condition (3.7).

We first ran the pseudospectral model using (3.7) to determine the initial stream function. Choosing $C_{3}$ so that $|\zeta(\cdot, 0)|_{\infty}=1,|D \mathbf{u}|_{\infty} \approx .6$ and we expect that we will need $N \geq 77$ in order to compute the solution correctly. Figure 1 is a contour plot of $\zeta$ at $t=20$ for the case $N=32$. The contour interval is $10^{-1}$. The large amplitude in high wave numbers in small areas is typical of cases with insufficient resolution. Figures $2 \mathrm{a}$ and $2 \mathrm{~b}$ are contour plots of $\zeta$ at $t=20$ for the cases $N=64$ and $N=128$, respectively. The contour interval is .09 in both plots. As can be seen from the plots, we essentially have the correct solution at $N=64$, which is near the point predicted by the estimate (3.2). Figures $3 \mathrm{a}$ and $3 \mathrm{~b}$ show the corresponding solutions at $t=200$. The contour interval is .03 in both plots. Although the contour plots for the resolutions $N=64$ and $N=128$ appear identical, the contours for 


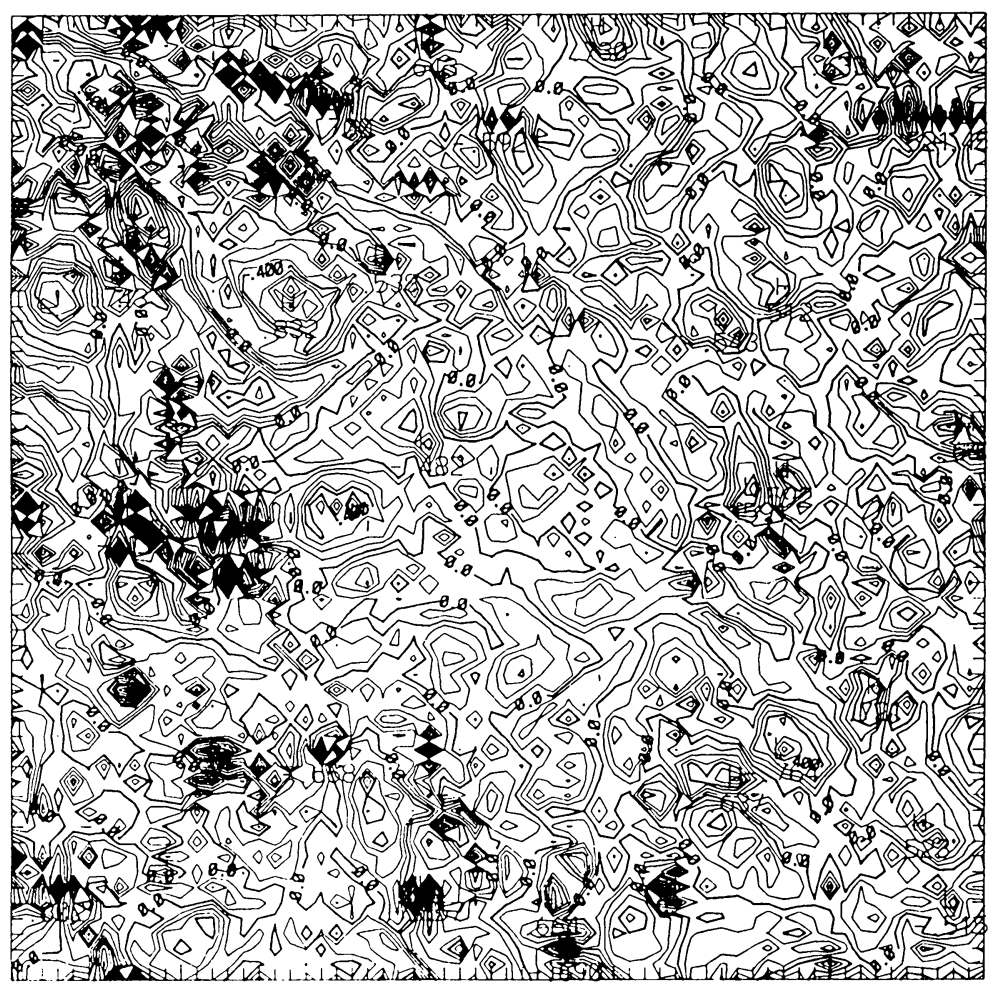

FIGURE 1

The vorticity $\varsigma$ at $t=20$ computed by the pseudospectral model $\left(\nu=10^{-4}, N=32\right)$ from the initial conditions (3.7). The contour interval is $1 \times 10^{-1}$.

the lower resolution are actually slightly shifted from those of the higher resolution. We also ran the case $N=80$, and then the contours are exactly aligned. To check the effect of the time truncation error, we also ran a case with $N=96$ and $\Delta t$ half the value for the case $N=128$. The contours were again exactly aligned, so that the temporal truncation error is not affecting the solution. This is surprising, as one might at first think that the temporal truncation error would dominate. We shall discuss this in the section on difference methods.

The estimate (3.2) only depends on the kinematic viscosity $\nu$. For $\nu=10^{-4}$, we also obtained convergence of the pseudospectral model for solutions resulting from the initial conditions determined by (3.5), (3.6), and (3.8). The fact that the numerical model converges to a solution gives us a base on which to test a number of previous analytic theories and numerical methods. We will perform these studies in the remaining sections.

The convergence of the numerical model indicates that the solution is not sensitive to small perturbations. As a further test of the effect of perturbations on the solution, we computed the initial stream function coefficients according to (3.7) and then added to each coefficient a term of exactly the same form but with .01 of the amplitude and a different random phase. The normalization $|\zeta(\cdot, 0)|_{\infty}=1$ 


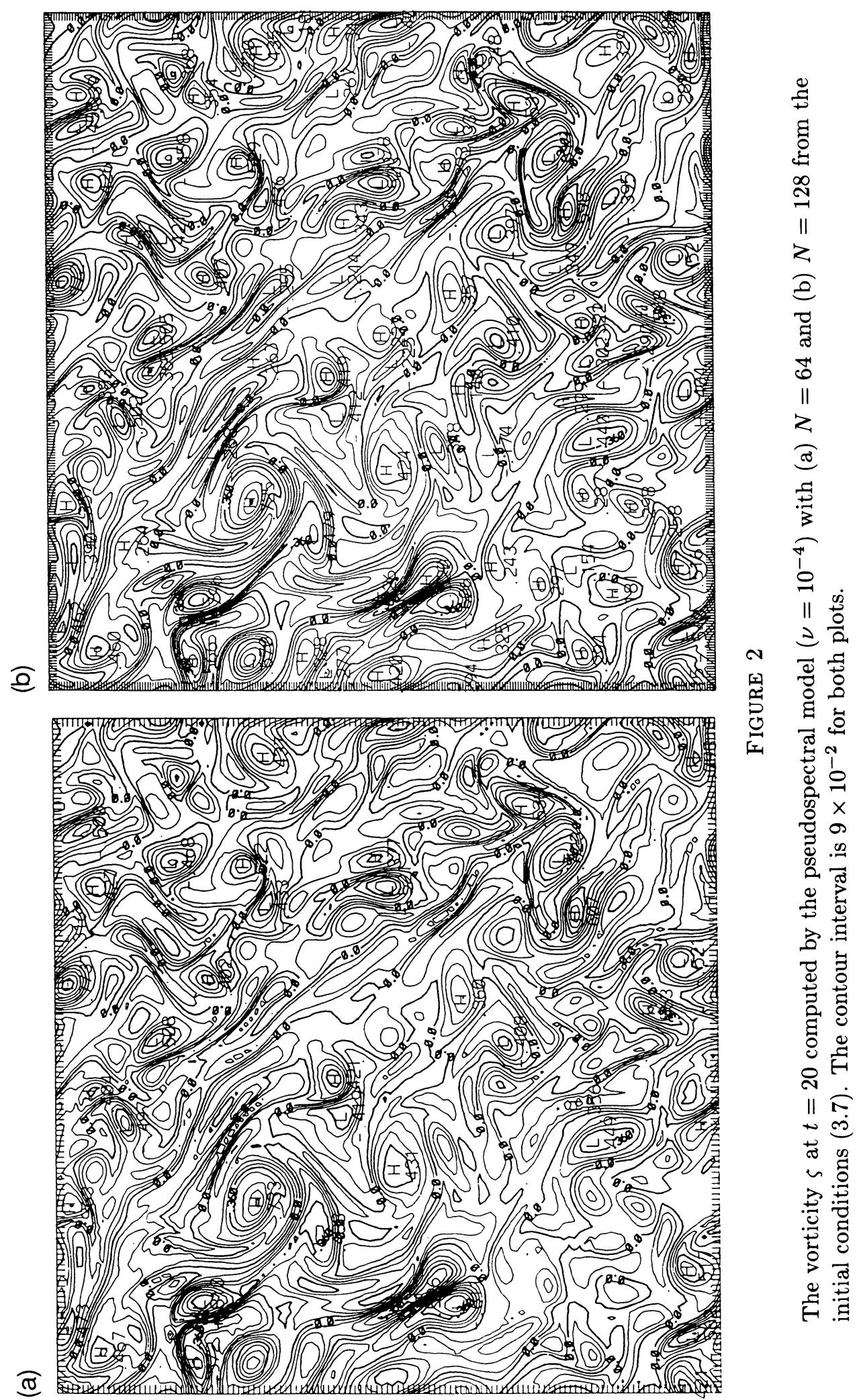




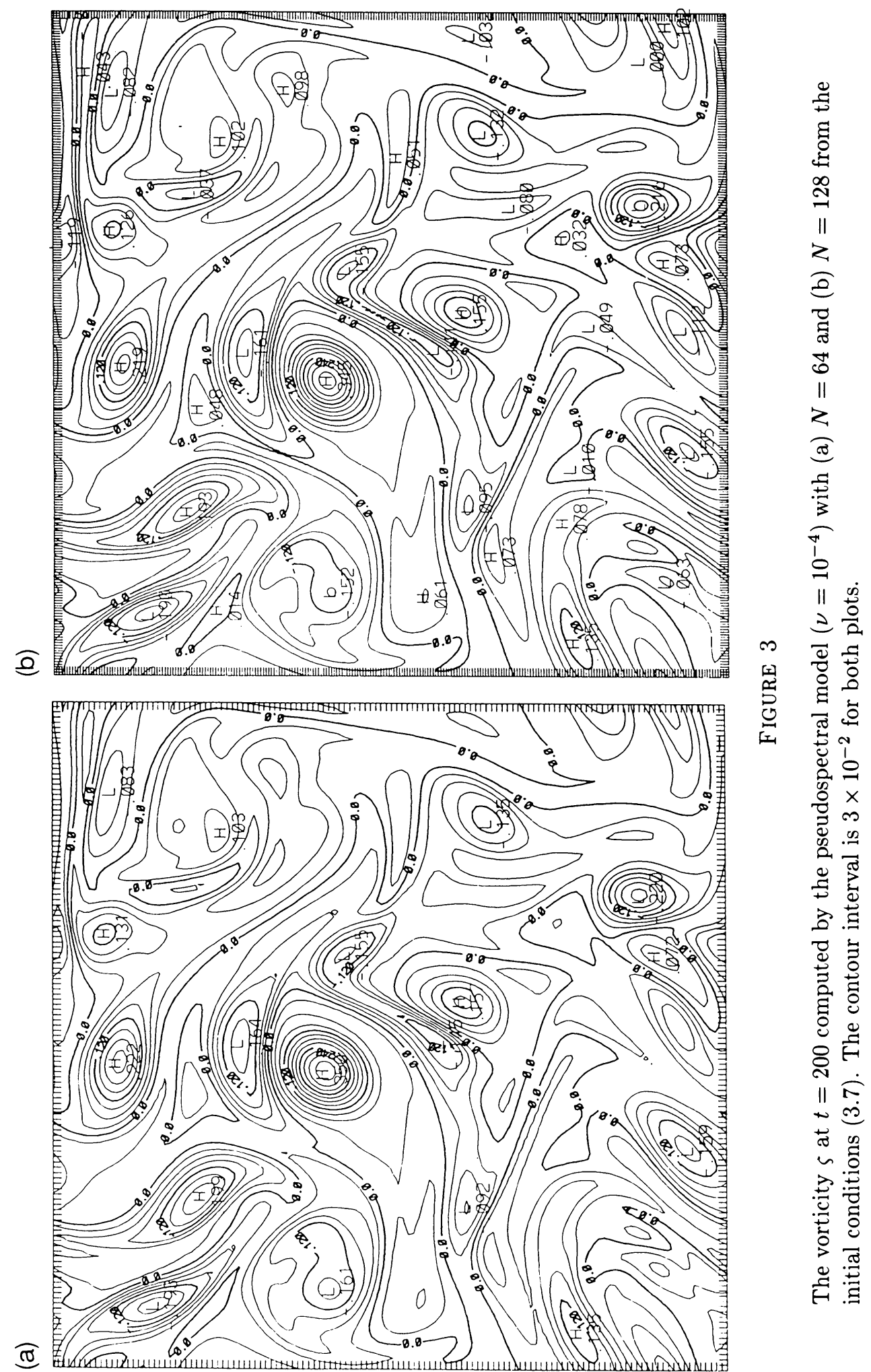




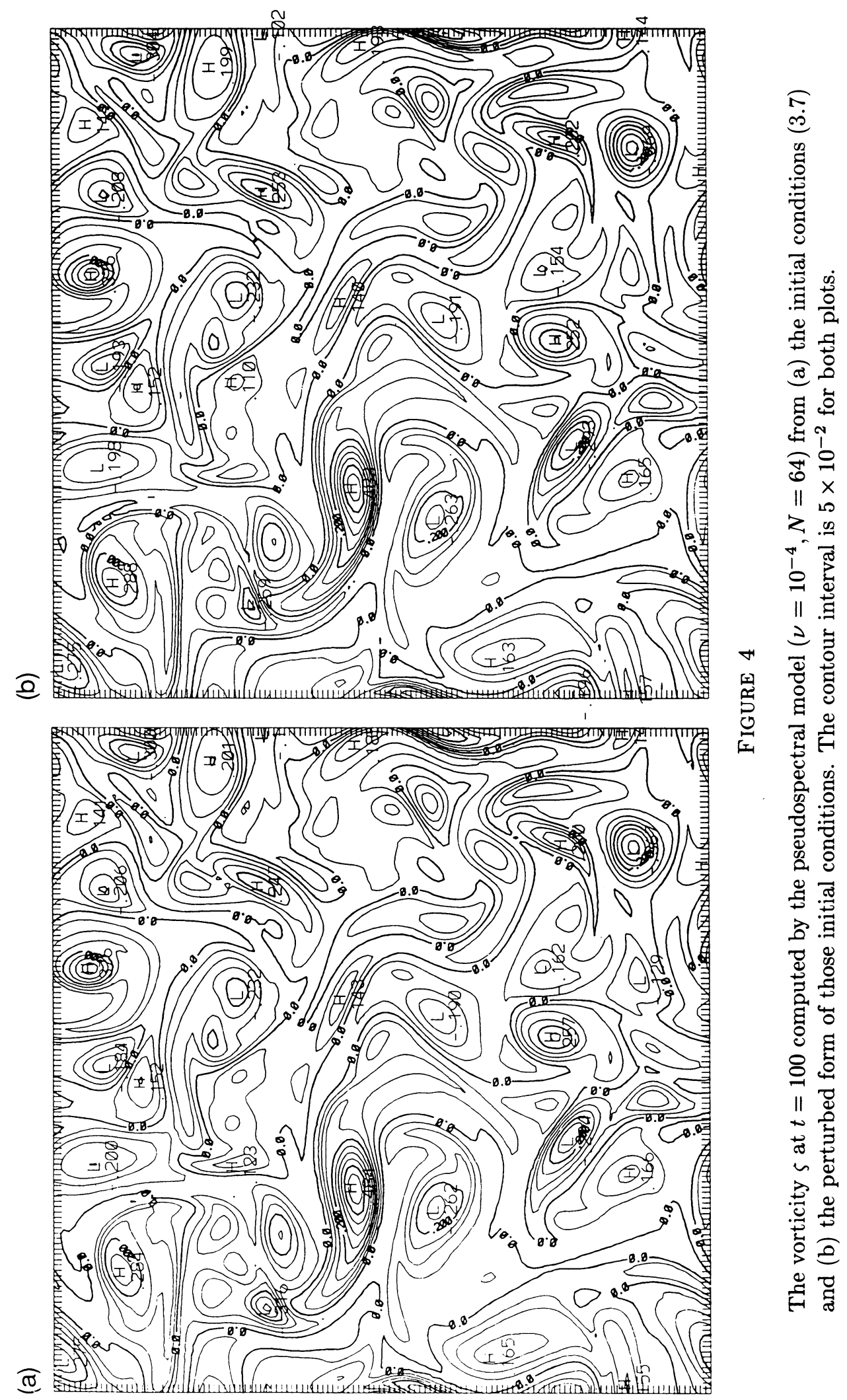




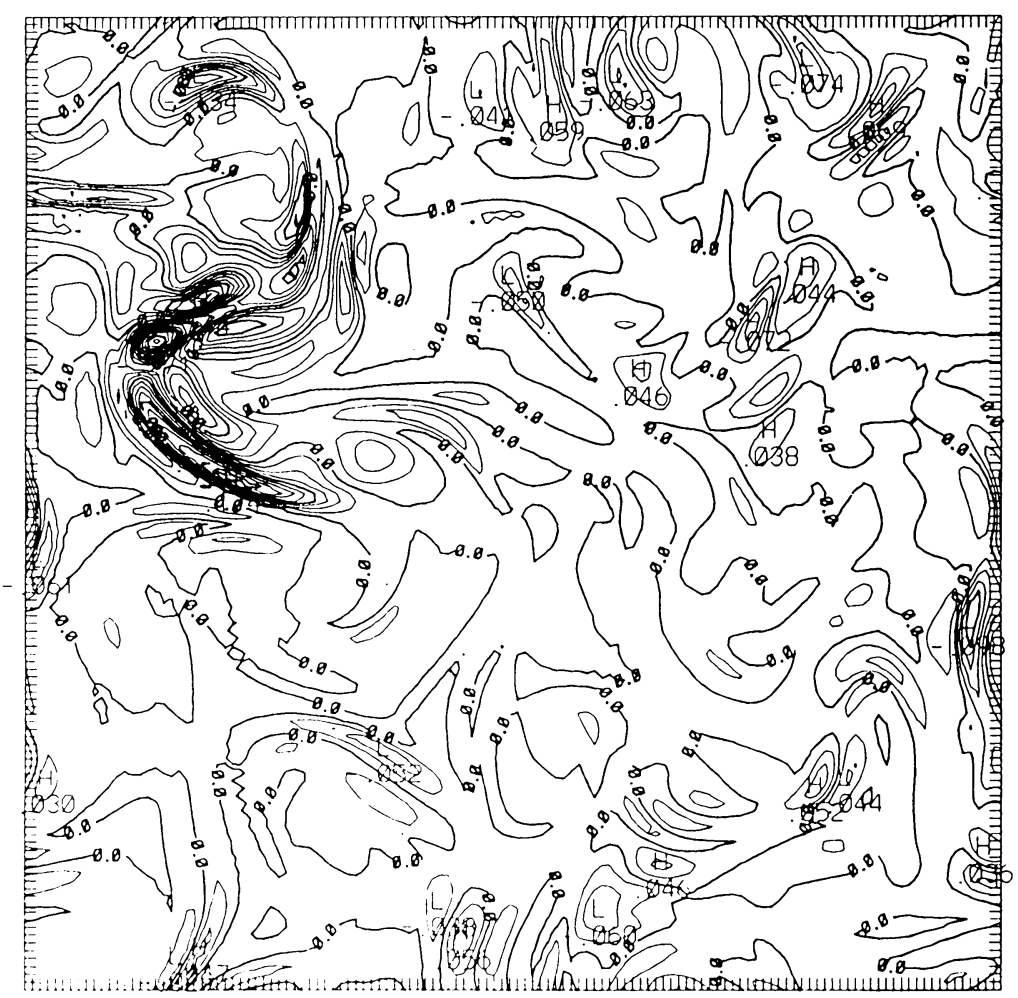

FIGURE 5

The difference map of the two plots in Figure 4. The contour interval is $2 \times 10^{-2}$.

was carried out after the addition of the perturbation. Figures $4 \mathrm{a}$ and $4 \mathrm{~b}$ are plots at $t=100$ of the solutions resulting from the unperturbed and perturbed initial conditions (3.7). The contour interval is .05 in both plots. It is clear that the two solutions are quite similar, even this far in the integration. The relative $L_{2}$ and $L_{\infty}$ errors at this time are .22 and .56 , respectively. To better determine the nature of the error, in Figure 5 the difference between the two solutions is plotted. The contour interval is .02 for this plot. As can be seen from the difference map, the largest errors occur in the narrow sheets where the gradients are large. This general pattern is evident throughout the integration.

4. Chopping. A number of models have set the amplitudes of all Fourier coefficients above a given wave number to zero at regular intervals as a mechanism to dissipate the energy in the high wave numbers. This method is commonly referred to as chopping the spectrum. Usually, chopping is used to stabilize a model that has insufficient resolution, i.e, if we used the correct viscosity coefficient the model would blow up. Fornberg [4] used chopping in place of the normal viscosity in his study of two-dimensional turbulence. In his study he tried chopping the spectrum at different intervals. We have run the pseudospectral model using chopping instead of viscosity on several of the initial conditions given in Section 3. In Figures 6a and $6 \mathrm{~b}$ we show the solution obtained from the initial condition (3.7) at $t=20$ 
and $t=200$, respectively. The contour interval is .1 for both plots. In this case we have chopped the upper third of the spectrum every 60 time steps. We also ran the model with chopping intervals of 15,30 , and 120 with similar results. As can be seen from the plots, the solution at $t=20$ is similar to the correct solution shown in Figure $2 \mathrm{a}$ but has considerably more structure. At $t=200$ the solution is completely different from the correct solution shown in Figure $2 \mathrm{~b}$. Thus, for short-term integrations the solution is not very sensitive to the chopping interval, but the solution is not very accurate.

If we run the chopping model on initial conditions whose Fourier amplitudes are not zero above the chosen chopping wave number (and this would normally be the case for general initial conditions), then the effect of the chopping on a short-term integration is much more serious. Figure $7 \mathrm{a}$ is a plot of the converged solution at $t=20$ using $\nu=10^{-4}$ and the initial condition

$$
|\hat{\psi}|=C_{3}\left\{|\mathbf{k}|^{-2}\left[1+(|\mathbf{k}| / 6)^{4}\right]^{-1}\right\}^{1 / 2}, \quad|\mathbf{k}|>0 .
$$

Figure $7 \mathrm{~b}$ shows the corresponding chopping solution at $t=20$. The contour intervals are .06 and .1 in Figure $7 \mathrm{a}$ and Figure $7 \mathrm{~b}$, respectively. Clearly, the chopping solution in this case is much worse.

From the comparisons above we see that whether or not chopping gives approximately the correct solution depends on the initial conditions and the length of integration. Thus we feel that the method is not very robust. However, we have only used the method for $\nu=10^{-4}$. It is possible that for smaller values of $\nu$ the results might be better.

5. Hyperviscosity. A number of modelers have used alternate forms of dissipation to remove the energy from higher wave numbers in a more selective fashion, i.e., there is less damping of low wave numbers and more damping of high wave numbers as compared to the normal viscosity. For example, McWilliams [10] has used the square of the Laplacian, which he calls hyperviscosity, in place of the Laplacian, so that he uses the system

$$
\begin{aligned}
& \varsigma_{t}+u \varsigma_{x}+v \zeta_{y}=-\nu \nabla^{4} \varsigma \\
& \nabla^{2} u=-\varsigma_{y} \\
& \nabla^{2} v=\varsigma_{x}
\end{aligned}
$$

instead of (2.4). In this section we want to apply the analysis used by Henshaw et al. [6] to (5.1) to understand the impact of the modification of the form of the dissipation.

In the case of (5.1) the estimate corresponding to (3.1) is

$$
\sup _{t \geq 1}|\hat{\zeta}| \leq\left[\frac{K_{q}(\alpha)|D \mathbf{u}|_{\infty}^{1 / 4+\alpha}}{\nu^{1 / 4+\alpha}|\mathbf{k}|}\right]^{2 q}
$$

We shall indicate the proof. Let

$$
(f, g)=\int_{R} f g d x d y, \quad\|f\|^{2}=(f, f),
$$

denote the (real) $L_{2}$ scalar product and norm, and use the notation

$$
J_{p}^{2}(t)=\left\|\partial^{p} \zeta(\cdot, t) / \partial x^{p}\right\|^{2}+\left\|\partial^{p} \zeta(\cdot, t) / \partial y^{p}\right\|^{2} .
$$



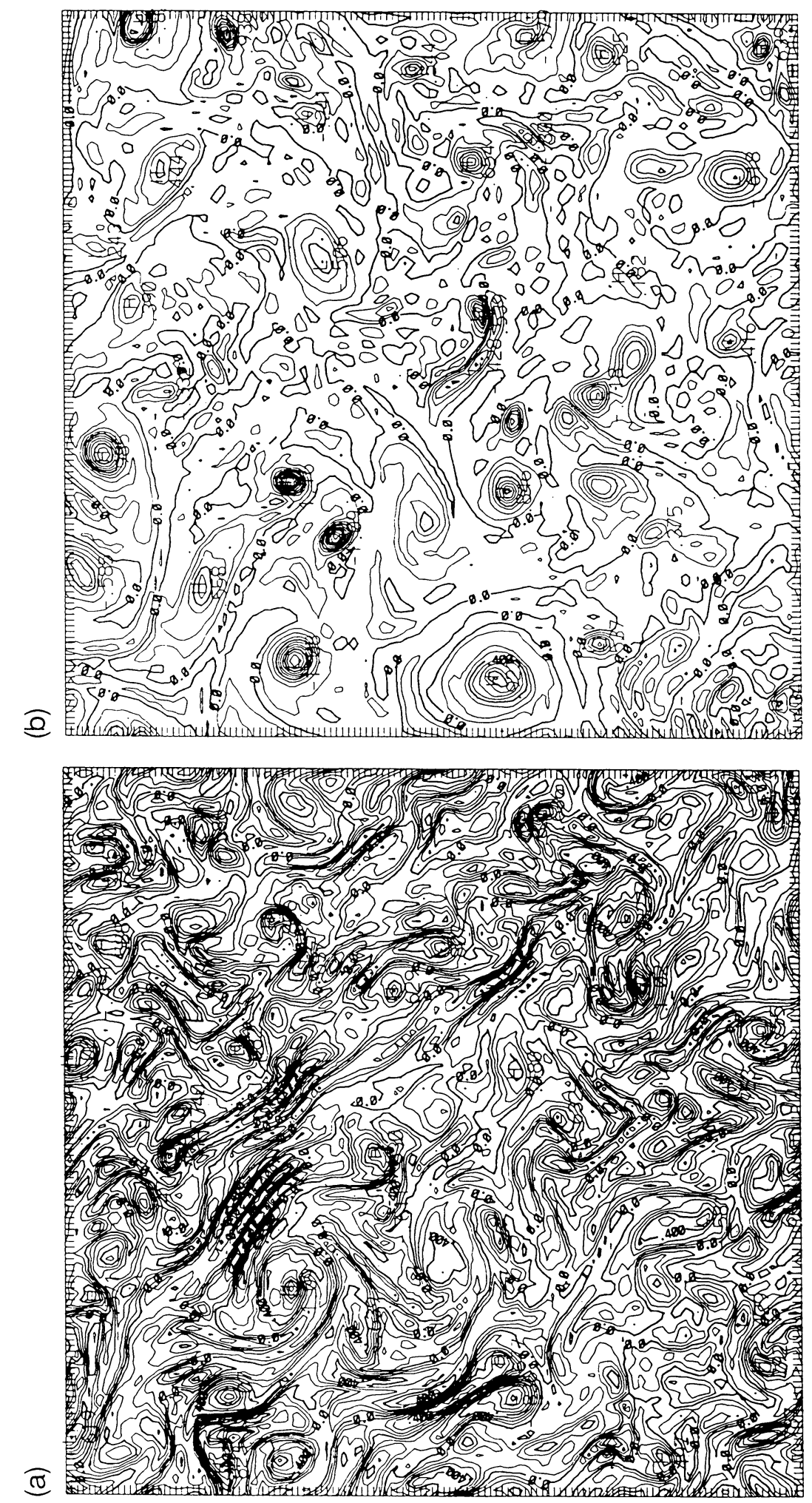

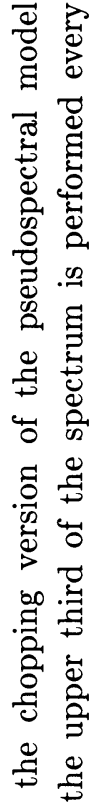

0 है

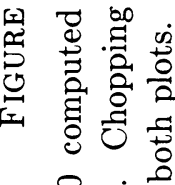

유:

ตृष्

11 T

+ 뭉으

อ

응 용

สี

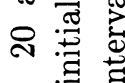

$\| \stackrel{\Xi}{. \Xi}$

↔先

ङ

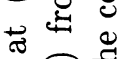

थ नु है

总

○े

量 11 

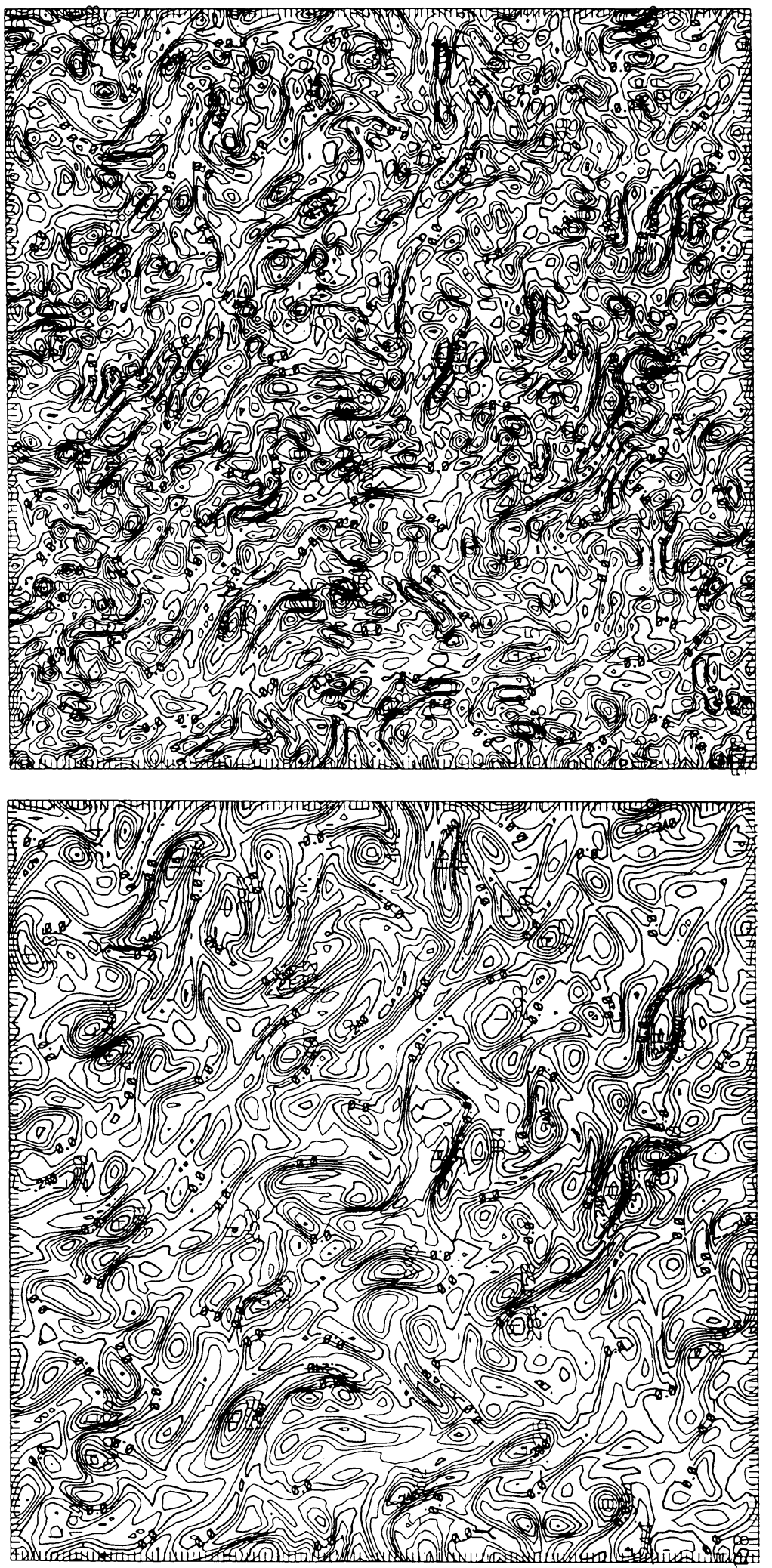

苍

$>\pi$

我 $\frac{2}{2}$

荅范

잉. 을

폰

ฟ

อ

ช

สี

उु०

11 .

之 ।

* 의의

I

II

$\lambda$ ฮ

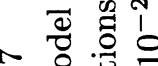

욤

괼 운

:

工范

造 売

옹

品

()

$\infty$

肎

ठิ 11

Z

总

द्व 11 웜

व्. $\supset$ क

ㅇㅝㅁㅀㅁ

11 छ 8

䒕

的范

خे

헝

?

$>$ ڤ

¿

F 공 
Integration by parts gives us

$$
\|\zeta\|_{t}^{2}=-2 \nu\left\|\nabla^{2} \varsigma\right\|^{2} \leq-2 \nu J_{2}^{2} \leq 0
$$

Therefore,

$$
\|\zeta(\cdot, t)\|^{2} \leq\|\zeta(\cdot, 0)\|^{2}, \quad 2 \nu \int_{0}^{\infty} J_{2}^{2} d t \leq\|\zeta(\cdot, 0)\|^{2} .
$$

Let $D^{p_{\zeta}}=\partial^{p_{\zeta}} / \partial x^{p_{1}} \partial y^{p_{2}}, p=p_{1}+p_{2}$, denote a general derivative of order $p$. From (5.1a),

$$
\frac{\partial}{\partial t}\left(t^{p}\left\|D^{2 p}\right\|^{2}\right)=p t^{p-1}\left\|D^{2 p} \varsigma\right\|^{2}-2 t^{p} \mathrm{I}+2 \nu t^{p}\left(D^{2 p} \varsigma, \nabla^{4} D^{2 p} \varsigma\right)
$$

where

$$
\mathrm{I}=\left(D^{2 p}{ }_{\zeta} D^{2 p}\left(u \zeta_{x}\right)+D^{2 p}\left(v \zeta_{y}\right)\right) .
$$

For $p=1$, we obtain

$$
\mathrm{I}=\mathrm{II}+2 \mathrm{III}, \quad \mathrm{II}=\left(D^{2}{ }_{\zeta},\left(D^{2} u\right)_{\zeta_{x}}+\left(D^{2} v\right)_{\zeta_{y}}\right), \quad \mathrm{III}=\left(D^{2}{ }_{\zeta} D u D \zeta_{x}+D v D \zeta_{y}\right),
$$

and integration by parts gives us

$$
\begin{aligned}
|\mathrm{II}| & =\left|\left(D_{\zeta}, D^{3} u \zeta_{x}+D^{3} v \zeta_{y}\right)\right| \\
& \leq \frac{|\zeta|_{\infty}}{2}\left(\left\|D_{\zeta_{x}}\right\|^{2}+\left\|D_{\zeta_{y}}\right\|^{2}+\left\|D^{3} u\right\|^{2}+\left\|D^{3} v\right\|^{2}\right) \\
|\mathrm{III}| & \leq \frac{|D \mathbf{u}|_{\infty}}{2}\left(\left\|D_{\zeta_{x}}\right\|^{2}+\left\|D_{\zeta_{y}}\right\|^{2}+2\left\|D^{2}\right\|^{2}\right) .
\end{aligned}
$$

Fourier analysis tells us that

$$
\begin{aligned}
& \left\|u_{x x x}\right\|^{2}+\left\|u_{y y y}\right\|^{2}+\left\|v_{x x x}\right\|^{2}+\left\|v_{y y y}\right\|^{2} \leq J_{2}^{2} \\
& \left\|\zeta_{x x}\right\|^{2}+2\left\|\zeta_{x y y}\right\|^{2}+\left\|\zeta_{y y}\right\|^{2} \leq 2 J_{2}^{2} .
\end{aligned}
$$

Also,

$$
|s|_{\infty} \leq 2|D \mathbf{u}|_{\infty}
$$

Therefore,

$$
\frac{\partial}{\partial t}\left(t J_{2}^{2}\right) \leq J_{2}^{2}+12 t|D \mathbf{u}|_{\infty} J_{2}^{2}-2 \nu t J_{4}^{2}
$$

Thus,

$$
T J_{2}^{2} \leq \int_{0}^{T} J_{2}^{2} d t+12 T|D \mathbf{u}|_{\infty} \int_{0}^{T} J_{2}^{2} d t-2 \nu \int_{0}^{T} t J_{4}^{2} d t
$$

i.e., by (5.4),

$$
\begin{gathered}
J_{2}^{2} \leq \frac{12 T|D \mathbf{u}|_{\infty}+1}{2 \nu T}\left\|_{\zeta}(\cdot, 0)\right\|^{2}=\left[\frac{6|D \mathbf{u}|_{\infty}}{\nu}+O(1 / T)\right]\|\zeta(\cdot, 0)\|^{2} \\
2 \nu \int_{0}^{T} t J_{4}^{2} d t \leq\left(12 T|D \mathbf{u}|_{\infty}+1\right) \int_{0}^{T} J_{2}^{2} d t
\end{gathered}
$$

For $p=2$ we have

$$
\mathrm{I}=\mathrm{II}+\mathrm{III}
$$




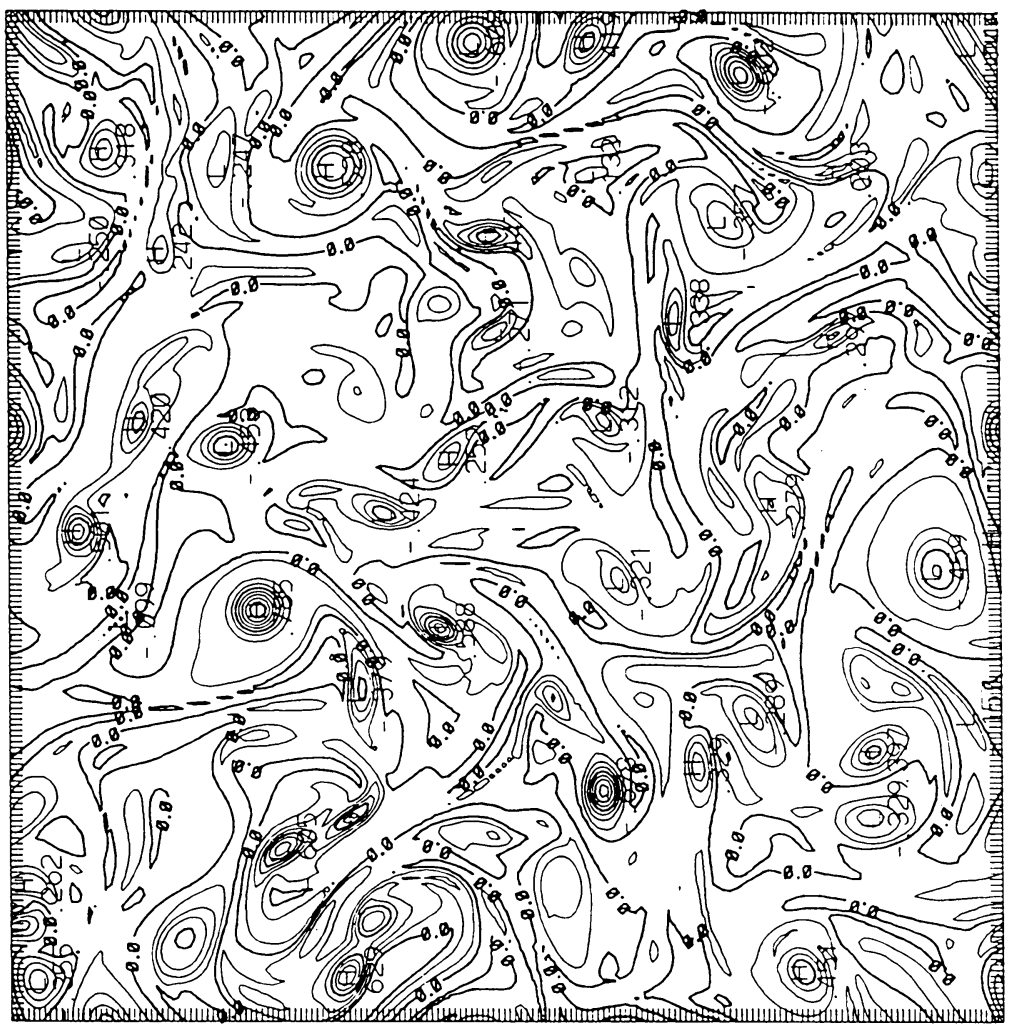

으.

(7)

ชิ.

คิ

$\|$ כ̄

$z$

$i \infty$

으

$\| \dot{\Xi}$

ก تี

क्ष

द

तี

芇

总.

을

己ِ

a

$\infty$ :

된

象

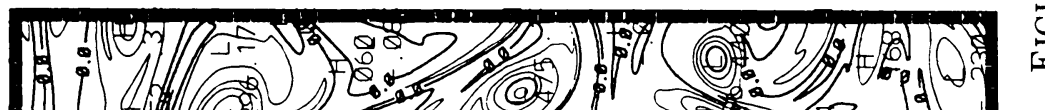

II

$\frac{\tilde{\pi}}{3} z$

18. (1) D.

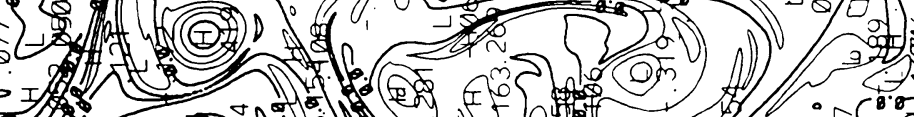
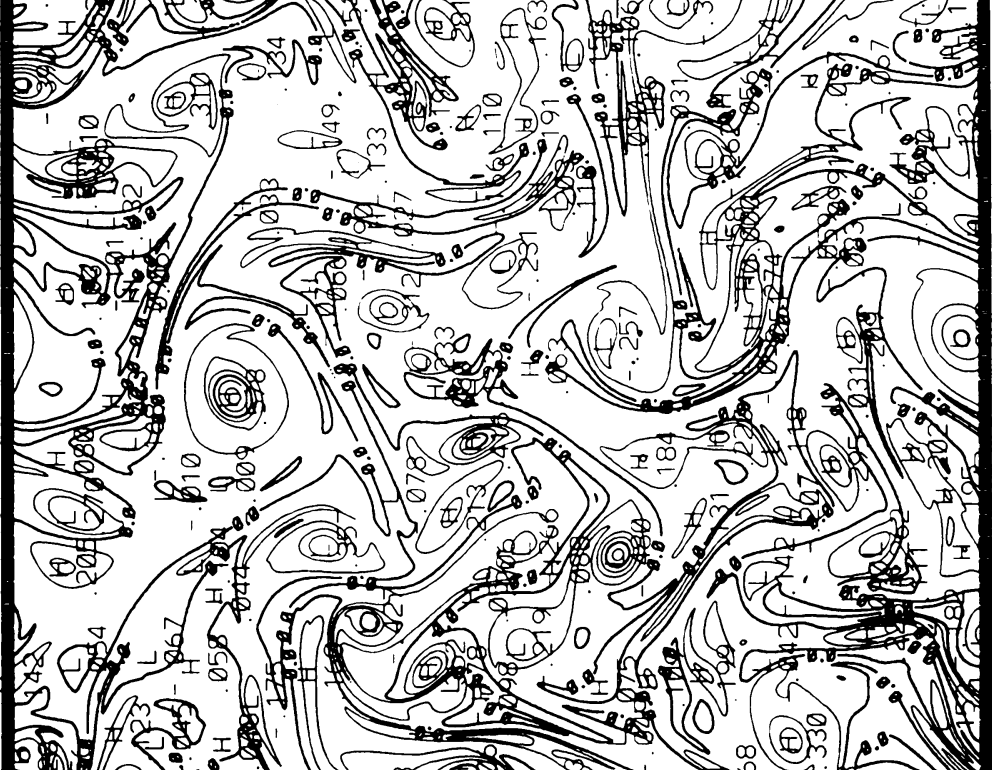

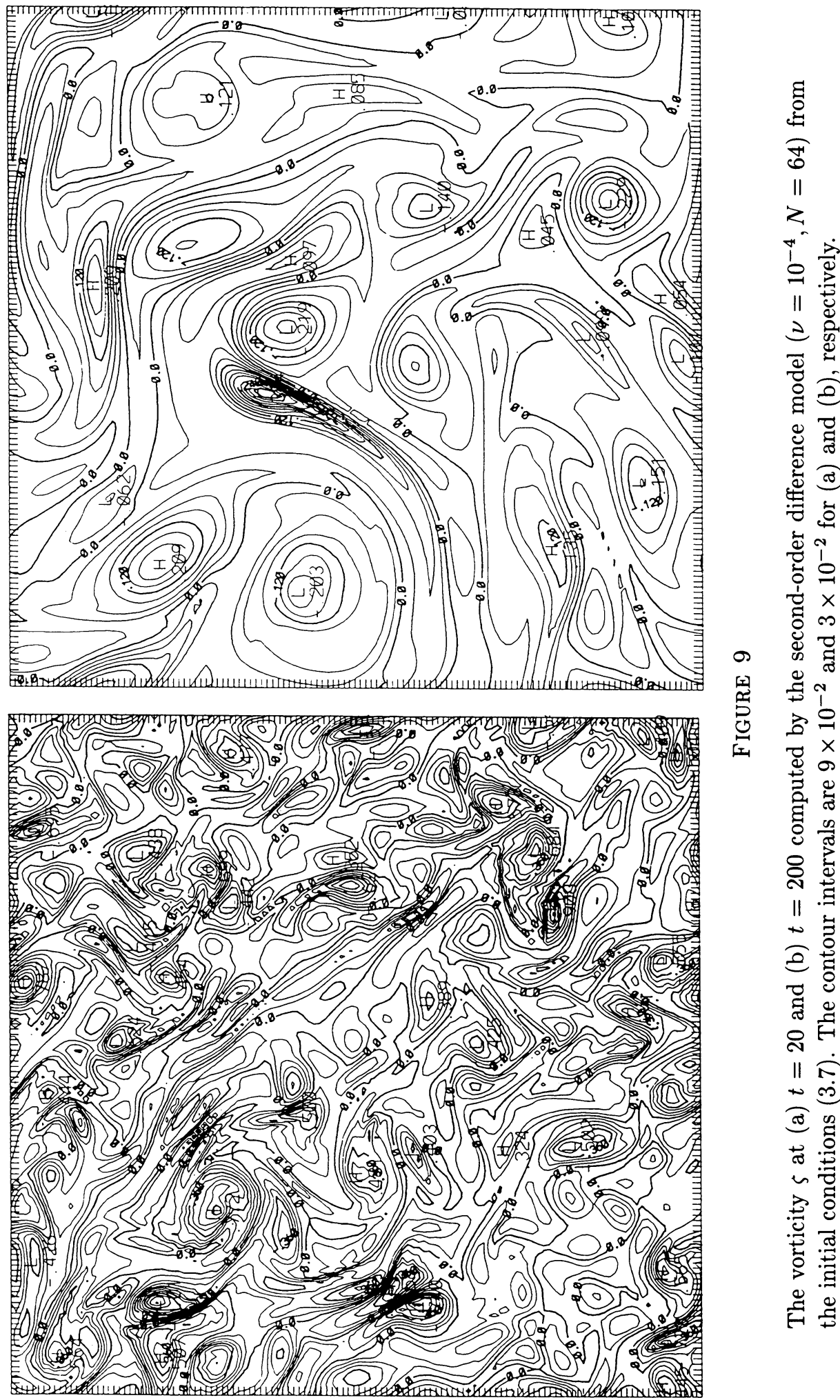
where for any $\gamma_{j}>0$

$$
\begin{aligned}
& |\mathrm{II}|=\left|\left(D^{4}{ }_{\zeta},\left(D^{4} u\right) \zeta_{x}+\left(D^{4} v\right) \zeta_{y}\right)+\left(D^{4}{ }_{\zeta}, D^{3} u\left(D_{\zeta_{x}}\right)+D^{3} v\left(D_{\zeta_{y}}\right)\right)\right| \\
& =\left|\left(D^{5} \varsigma,\left(D^{3} u\right) \zeta_{x}+\left(D^{3} v\right) \zeta_{y}\right)\right| \\
& \leq \frac{\nu \gamma_{1}}{2}\left(\left\|D^{5} \zeta_{x}\right\|^{2}+\left\|D^{5} \zeta_{y}\right\|^{2}\right)+\frac{1}{2 \nu \gamma_{1}}|\zeta|_{\infty}^{2}\left(\left\|D^{3} u\right\|^{2}+\left\|D^{3} v\right\|^{2}\right), \\
& |\mathrm{III}|=\left|\left(D^{4}{ }_{\zeta},\left(D^{2} u\right) D^{2} \zeta_{x}+\left(D^{2} v\right) D^{2} \varsigma_{y}\right)+\left(D^{4}{ }_{\zeta},(D u) D^{3}{ }_{\zeta_{x}}+(D v) D^{3} \zeta_{y}\right)\right| \\
& =\left|\left(D^{5}{ }_{\zeta},(D u) D^{2} \zeta_{x}+(D v) D^{2} \varsigma_{y}\right)\right| \\
& \leq \frac{\nu \gamma_{2}}{2}\left(\left\|D^{5} \zeta_{x}\right\|^{2}+\left\|D^{5} \varsigma_{y}\right\|^{2}\right)+\frac{|D \mathbf{u}|_{\infty}^{2}}{\nu \gamma_{2}}\left\|D^{2} \varsigma\right\|^{2} \text {. }
\end{aligned}
$$

Thus,

$$
\frac{\partial}{\partial t}\left(t^{2} J_{4}^{2}\right) \leq 2 t J_{4}^{2}-t^{2}\left(\frac{2}{\nu \gamma_{1}}+\frac{1}{\nu \gamma_{2}}\right)|D \mathbf{u}|_{\infty}^{2} J_{2}^{2}-t^{2}\left[2 \nu-\frac{\nu\left(\gamma_{1}+\gamma_{2}\right)}{2}\right] J_{6}^{2} .
$$

For $\gamma_{1}=\gamma_{2}=1$ we obtain from (5.4) and (5.5)

$$
\begin{aligned}
T^{2} J_{4}^{2}(T) & \leq \int_{0}^{T}\left(2 t J_{4}^{2}\right) d t+\frac{3|D \mathbf{u}|_{\infty}^{2}}{\nu} \int_{0}^{T} t^{2} J_{2}^{2} d t \\
& \leq \frac{\left(8 T|D \mathbf{u}|_{\infty}+1\right)}{4 \nu^{2}}\left\|_{\zeta}(\cdot, 0)\right\|^{2}+\frac{3 T^{2}}{2 \nu^{2}}|D \mathbf{u}|_{\infty}^{2}\left\|_{\zeta}(\cdot, 0)\right\|^{2}
\end{aligned}
$$

i.e.,

$$
J_{4}^{2}(T) \leq\left[\frac{3|D \mathbf{u}|_{\infty}^{2}}{2 \nu^{2}}+O(1 / T)\right]\|\varsigma(\cdot, 0)\|^{2}
$$

Therefore, we can also estimate $\nu \int_{0}^{T} t^{2} J_{6}^{2} d t$ by the right-hand side of (5.6).

This process can be continued for $p=3,4$, and we obtain the bounds

$$
J_{2 p}^{2}(T) \leq\left[K_{p}\left(\frac{|D \mathbf{u}|_{\infty}}{\nu}\right)^{p}+O(1 / T)\right]\|\zeta(\cdot, 0)\|^{2} .
$$

For $p>4$ the estimates become slightly more complicated. However, as in Henshaw et al. [6], we can prove

$$
J_{2 p}^{2}(T) \leq\left[K_{p}(\alpha)\left(\frac{|D \mathbf{u}|_{\infty}}{\nu}\right)^{p+\alpha}+O(1 / T)\right]\|\zeta(\cdot, 0)\|^{2}
$$

By Parseval's relation,

$$
|\hat{\zeta}(T)|^{2}|\mathbf{k}|^{4 p} \leq \text { const } J_{2 p}^{2}(T)
$$

and therefore the desired inequality follows. In view of (5.4), (5.5), and (5.6), for practical purposes we can again choose $\alpha=0$ and $K_{p}(\alpha)=1$.

The main reason for using hyperviscosity instead of regular viscosity is a desire to compute a solution with a smaller kinematic viscosity coefficient. This raises the question as to what hyperviscosity coefficient to choose to obtain the same solution as for the regular viscosity case. A reasonable constraint on the coefficient of the hyperviscosity is given by the condition that the spectrum using hyperviscosity should become negligible at the same wave number as when using normal viscosity, i.e.,

$$
|D u|_{\infty}^{1 / 4} \nu_{4}^{-1 / 4}=|D u|_{\infty}^{1 / 2} \nu_{2}^{-1 / 2}
$$




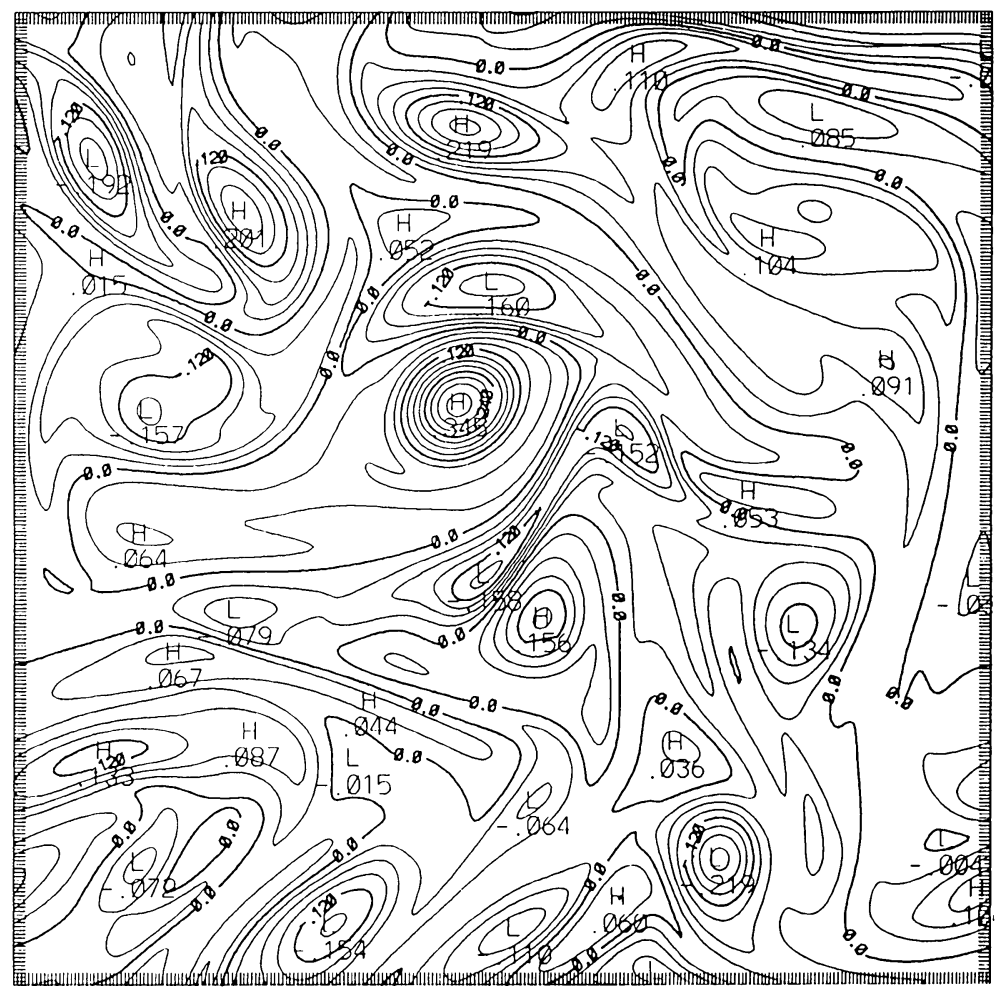

FIGURE 10

The vorticity $\varsigma$ at $t=200$ computed by the second-order difference model $\left(\nu=10^{-4}, N=128\right)$ from the initial conditions (3.7). The contour interval is $3 \times 10^{-2}$.

where $\nu_{2}$ and $\nu_{4}$ are the coefficients of the normal and hyperviscosity, respectively. For a kinematic viscosity coefficient $\nu_{2}=10^{-5}$ this means we should use a hyperviscosity coefficient $\nu_{4} \approx 10^{-10}$. When we used this value for the hyperviscosity coefficient, the solution was completely different from the regular viscosity solution. Thus we were forced to try different values of $\nu_{4}$. Figures $8 \mathrm{a}$ and $8 \mathrm{~b}$ are plots of the vorticity $\varsigma$ at $t=200$ using the initial conditions (4.1) for the regular viscosity $\left(\nu_{2}=10^{-5}\right)$ and hyperviscosity $\left(\nu_{4}=10^{-8}\right)$ version of the pseudospectral model, respectively. The contour interval is .09 in both plots. As the results show, the general features of the solution (vortex sheets, roll up, and vortex blobs) are similar. However, the actual details are quite different. It is possible to have either the blob amplitude or spatial extent similar, but not both. These differences are more pronounced for larger values of $\nu_{2}$. Thus it is conceivable that this difference would disappear for even smaller values of the viscosity coefficients. However, then the number of waves has to be increased considerably and none of the existing calculations have sufficient resolution. Also, there is always a period when the flow is maximal dissipative, i.e., $\nu_{2} J_{1}^{2}=O(1)$ or $\nu_{4}^{2} J_{2}^{2}=O(1)$, and it is during this time that the difference is created. Therefore, it seems to be unlikely that the difference between the solutions will disappear as the viscosity coefficients approach zero. 


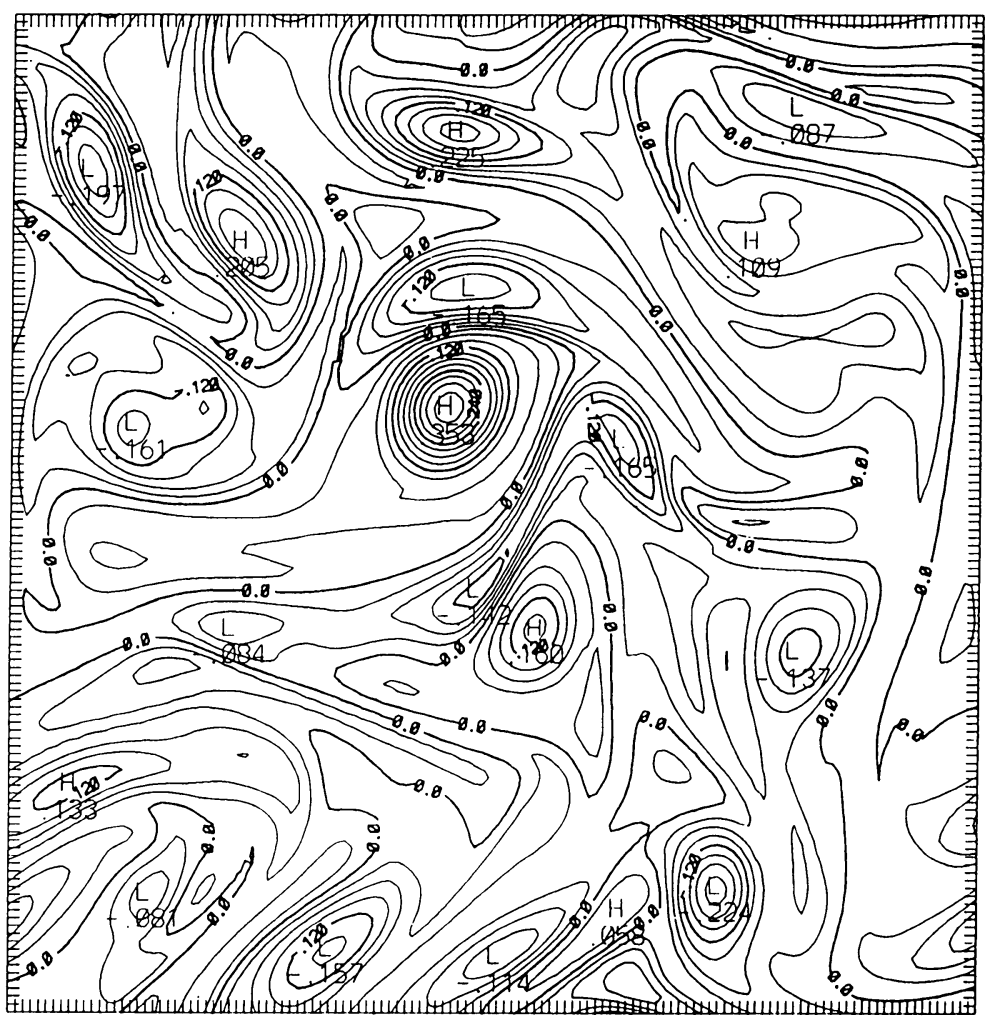

FIGURE 11

The vorticity $\zeta$ at $t=200$ computed by the fourth-order difference model $\left(\nu=10^{-4}, N=64\right)$ from the initial conditions (3.7). The contour interval is $3 \times 10^{-2}$.

6. Difference Methods. Many investigations of two-dimensional turbulence have been based on finite difference models (Lilly [9], Deem and Zabusky [3]). More recently, the pseudospectral method has become popular (e.g., Fox and Orszag [5]). For a given set of initial conditions we want to compare solutions computed by second- and fourth-order finite difference methods with the solution computed by the pseudospectral method on the same grid. Figures $9 \mathrm{a}$ and $9 \mathrm{~b}$ are plots of the vorticity at $t=20$ and $t=200$, respectively, obtained from the second-order finite difference model $\left(\nu=10^{-4}, N=64\right)$ using the initial conditions (3.7). The contour intervals are the same as those in Figures 2a and 3a for comparison with those plots. The second-order finite difference solution is close to that of the pseudospectral solution at $t=20$, but at $t=200$ they are completely different. Thus, if computations are only needed for a short period of time, the secondorder finite difference model is perfectly adequate, but for long periods of time it is not. We also ran the second-order model with twice the number of grid points $(N=128)$. Figure 10 shows the vorticity $\varsigma$ at $t=200$ for that case. With double the resolution of the spectral model, the second-order method produces a solution which is acceptable for this length of integration. 
Figure 11 is a plot of the vorticity at $t=200$ computed by the fourth-order finite difference model ( $\left.\nu=10^{-4}, N=64\right)$ using the initial conditions (3.7). The contour interval is the same as in Figure 3a. Clearly, the solutions are essentially identical, and this is also true for the case $\nu=10^{-5}$. It is clear from these comparisons that the fourth-order finite differences are sufficient to reduce the spatial error to a size which is totally acceptable, i.e., the fourth-order model is adequate for both shortand long-term integrations. We note that in the fourth-order model the diffusion term was lagged in time and only treated by a second-order approximation in space.

Perhaps it is surprising that the difference methods give such good results. This can be explained in the following manner. The first 15 wave numbers of the spectrum contain the majority of the energy of the solution. The fourth-order solution was calculated with $128 \times 128$ grid points. Therefore, there are enough points per wave length to resolve the flow (Kreiss and Oliger [8]). This also explains why the time integration does not cause any problems. We used the same time step for the finite difference methods as for the pseudospectral method. Since this is more than a factor of two times smaller than necessary for stability, the time error is reduced to an acceptable level similar to the case of the second-order difference method with twice the number of grid points.

National Center for Atmospheric Research

P.O. Box 3000

Boulder, Colorado 80307

E-mail: browning@silver.ucar.edu

Department of Mathematics

University of California, Los Angeles

Los Angeles, California 90024

1. D. L. BOOK, NRL Plasma Formulary, Naval Research Laboratory, 1980, 60 pp.

2. M. E. Brachet, M. MENeguzzi \& P. L. Sulem, "Small-scale dynamics of high-Reynoldsnumber two-dimensional turbulence," Phys. Rev. Lett., v. 57, 1986, pp. 683-686.

3. G. S. DEEM \& N. J. ZABUSKY, "Vortex waves: stationary $V$-states, interactions, recurrence and breaking," Phys. Rev. Lett., v. 40, 1978, pp. 859-862.

4. B. Fornberg, "A numerical study of 2-d turbulence," J. Comput. Phys., v. 25, 1977, pp. $1-31$.

5. D. G. Fox \& S. A. OrszAG, "Pseudospectral approximation to two-dimensional turbulence," J. Comput. Phys., v. 11, 1973, pp. 612-619.

6. W. D. HENSHAW, H.-O. KREISS \& L. G. REYNA, On the Smallest Scale for the Incompressible Navier-Stokes Equations, ICASE Report No. 88-8, 1988, 49 pp.

7. J. R. Herring, S. A. Orszag, R. H. Kraichnan \& D. G. Fox, "Decay of two dimensional turbulence," J. Fluid Mech., v. 66, 1974, pp. 417-444.

8. H. O. KREISS \& J. OLIGER, "Comparison of accurate methods for the integration of hyperbolic equations," Tellus, v. 24, 1972, pp. 199-215.

9. D. K. LiLLY, "Numerical simulation of developing and decaying of two-dimensional turbulence," J. Fluid Mech., v. 45, 1971, pp. 395-415.

10. J. C. MCWilliams, "The emergence of isolated coherent vortices in turbulent flow," $J$. Fluid Mech., v. 146, 1984, pp. 21-43. 